\title{
Perfil epidemiológico dos pacientes na lista de espera para transplante de córnea no Estado de Sergipe
}

\author{
Epidemiological profile of the patients on the waiting list for cornea transplantation in \\ the State of Sergipe, Brazil
}

\author{
Ângelo Auguusto Araújo ${ }^{1}$ \\ Gustavo Barreto de Melo ${ }^{2}$ \\ Renata Lemos Silva ${ }^{2}$ \\ Vitalina Martins de Araújo Neta ${ }^{2}$
}

\begin{tabular}{|l|}
\hline RESUMO \\
\hline Objetivo: Avaliar o perfil epidemiológico dos pacientes em lista de espera \\
e submetidos a transplante de córnea no estado de Sergipe e as principais \\
indicações para este procedimento. Métodos: Na Central de Transplantes \\
de Sergipe, foram colhidos os dados de 320 pacientes que entraram em lista \\
de espera edaqueles que tiveram a córnea transplantada entre maio de 2000 \\
e novembro de 2002. Os dados registrados foram: doença corneana, a \\
idade, o sexo, a acuidade visual e o tempo de espera na lista até a realização \\
do transplante. Resultados: A ceratopatia bolhosa do afácico e do pseu- \\
dofácico foi a doença mais freqüente (39,1\%), seguida por leucoma \\
(22,5\%), ceratocone (14,1\%), “outras doenças” (10,6\%), retransplante \\
(7,8\%) e úlcera (5,6\%). A idade média foi de 52,16 anos e não houve \\
diferença significante entre os sexos. A acuidade visual mais freqüente foi \\
visão de vultos e "percebe luz”. Os 110 pacientes que tiveram a córnea \\
transplantada esperaram, em média, seis meses. Os indicados por úlcera e \\
por retransplante tiveramprioridade, aguardando menos tempo. Os portado- \\
res de ceratopatia bolhosa encontravam-se na faixa etária média de 68,5 \\
anos e os com ceratocone, na de 23,6 anos. Conclusões: Pode-se concluir \\
que a ceratopatia bolhosa é a principal indicação para transplante de \\
córnea em nosso estado. Não há diferença entre a freqüência dos sexos; \\
aidade média da doença mais freqüente está de acordo com seu aparecimen- \\
to. A acuidade visual está dentro do esperado e o tempo em lista de espera \\
é longo e inadequado.
\end{tabular}

Descritores: Transplante de córnea; Perfis epidemiológicos; Ceratoplastia penetrante; Ceratocone; Epidemiologia

\section{INTRODUÇÃO}

A ceratoplastia penetrante (CP) figura, dentre as cirurgias de transplante, como o procedimento mais realizado ${ }^{(1)}$. A indicação para este tipo de cirurgia tem evoluído muito durante as últimas décadas. Isso se deve a diversos fatores, entre eles a melhor seleção do tecido doador e novas técnicas e equipamentos operatórios que permitem a realização de procedimentos cirúrgicos em situações consideradas inoperáveis há 10 anos $^{(2)}$. As estatísticas indicam que, nos Estados Unidos, são realizadas aproximadamente 35.000 transplantes de córnea anualmente ${ }^{(2)}$. A indicação mais comum varia muito quando analisados vários estudos, dependendo do período de tempo estudado, do método de coleta de dados, do local de obtenção dos dados e da população base de pacientes do cirurgião ${ }^{(2)}$.

De um modo geral, nos Estados Unidos, a principal indicação de CP ainda está relacionada a ceratopatia bolhosa do afácico e do pseudofácico 
(CBAP). Esses dados diferem quando comparados a publicações da Europa, Nova Zelândia e Brasil, onde a maioria dos relatos mostra o ceratocone como a indicação mais freqüente ${ }^{(3-9)}$. Analisando-se o perfil epidemiológico dessas duas doenças, pode-se dizer que a CBAP acomete predominantemente pacientes idosos, sendo 75,9 anos a média etária( ${ }^{(8,10)}$. Isso se explica pelo número elevado de pessoas que apresentam catarata e são submetidas à cirurgia. Já o ceratocone é uma doença que atinge principalmente jovens, em torno da terceira década de vida. Entretanto, é por volta dos 40 anos que a maioria dos pacientes realiza o procedimento cirúrgico ${ }^{(8,10)}$.

Este estudo avalia o perfil epidemiológico dos pacientes que têm indicação de transplante de córnea no estado de Sergipe, tendo como objetivo: definir as indicações mais freqüentes, cruzando com as seguintes variáveis: faixa etária média, sexo, tempo gasto na lista de espera e prioridades.

\section{MÉTODOS}

Foi realizado um estudo retrospectivo, analisando-se os dados existentes na Central de Transplantes de Sergipe, sediada no Hospital Governador João Alves Filho, durante o período de maio de 2000 até novembro de 2002. Neste intervalo de tempo, 320 olhos da mesma quantidade de pacientes entraram na lista. Destes, 110 foram submetidos ao transplante. Foram coletadas as seguintes variáveis: indicação, idade, sexo, acuidade visual pré-operatória e tempo decorrido desde a entrada na lista de espera até a realização do transplante.

As indicações foram divididas nas seguintes categorias: ceratopatia bolhosa do afácico e do pseudofácico com ou sem distrofia do endotélio corneano; ceratocone; leucoma de origem diversa; retransplante; úlcera corneana; outras doenças.

A acuidade visual pré-operatória foi dividida com base nos critérios estabelecidos pela Organização Mundial de Saúde (WHO), em seis categorias, sendo considerado:

$\begin{aligned} & \text { WHO } \text { AVL } \\ & \text { I - Melhor ou igual 20/200 } \\ & \text { II - Pior que 20/200 melhor que 20/400 } \\ & \text { III - Conta dedos } \\ & \text { IV - Vultos e percepção da luz (PL) } \\ & \text { V - Amaurose } \\ & \text { VI - Dados não disponíveis ou grande perda visual não } \\ &\end{aligned}$

As informações foram armazenadas em programa específico e analisadas estatisticamente pelo teste do Qui-quadrado para freqüências isoladas e de Kruskal-Wallis para comparar múltiplas variáveis, com índice de significância menor que 0,05.

\footnotetext{
* Foram mantidos os prontuários com acuidade visual não disponível ou com grandes perdas visuais não qualificadas devido ao grande número de cadastros observados em estudo piloto anteriormente realizado.
}

\section{RESULTADOS}

Ceratopatia bolhosa foi a doença mais encontrada entre as indicações para ceratoplastia penetrante na Central de Transplantes de Sergipe. Foi seguida por leucoma, ceratocone, outras doenças, retransplante e úlcera $\left(\chi^{2}=150,07\right.$ e p $\left.<0,001\right)$ (Tabela 1$)$.

Não houve diferença estatisticamente significante entre a freqüência dos sexos $\left(\chi^{2}=0,61\right.$ e $\left.p=0,434\right)$, sendo $52,2 \%$ do sexo masculino e $47,8 \%$ do feminino. A faixa etária média foi de 52,16 anos, sendo 1 ano a menor idade e 90 , a maior $\left(\chi^{2}=134,65\right.$ e $\mathrm{p}<0,001)$.

Visão de vultos e PL (IV) foi a acuidade visual mais freqüente, seguida por "não-disponível (VI)", conta dedos (III), “pior que 20/200 e melhor ou igual a 20/400 (II)", amaurose (V) e “melhor ou igual a 20/200 (I)” $\left(\chi^{2}=272,2\right.$ e p<0,001) (Tabela 2).

A média de tempo de espera dos 110 pacientes submetidos a transplante de córnea foi de 6,28 meses, sendo o mínimo de 1 dia e o máximo de 27 meses $\left(\chi^{2}=69,27\right.$ e $\left.p<0,001\right)$.

Os pacientes com ceratopatia bolhosa tinham, em média, 68,54 \pm 10 anos; os com ceratocone tinham faixa etária média de 23,66 \pm 12 ,9; os outros grupos de pacientes tinham idade intermediária $\left(\chi^{2}=140,3\right.$ e p<0,001) (Gráfico 1).

Os pacientes portadores de úlceras e com indicação de retransplante foram os que passaram menos tempo na lista de

\begin{tabular}{|c|c|c|}
\hline \multirow[b]{2}{*}{ Doença corneana } & \multicolumn{2}{|c|}{ Freqüência } \\
\hline & $\mathbf{n}$ & $\%$ \\
\hline Ceratopatia bolhosa & 125 & 39,1 \\
\hline Leucoma & 72 & 22,5 \\
\hline Ceratocone & 45 & 14,1 \\
\hline Outras doenças & 34 & 10,6 \\
\hline Retransplante & 25 & 7,8 \\
\hline Úlcera & 18 & 5,6 \\
\hline Não-disponível & 1 & 0,3 \\
\hline Total & 320 & 100,0 \\
\hline
\end{tabular}

\begin{tabular}{|c|c|c|}
\hline \multirow[b]{2}{*}{ Acuidade visual } & \multicolumn{2}{|c|}{ Freqüência } \\
\hline & $\mathrm{n}$ & $\%$ \\
\hline I (Melhor ou igual 20/200) & 21 & 6,6 \\
\hline II (Pior que 20/200 melhor que 20/400) & 26 & 8,1 \\
\hline III (Conta dedos) & 33 & 10,3 \\
\hline IV (Vultos e percepção da luz) & 160 & 50,0 \\
\hline V (Amaurose) & 23 & 7,2 \\
\hline $\begin{array}{l}\text { VI (Dados não disponíveis ou grande } \\
\text { perda visual não qualificada) }\end{array}$ & 57 & 17,8 \\
\hline Total & 320 & 100,0 \\
\hline
\end{tabular}


espera, com média, respectivamente, de 3,35 \pm 4,57 e 1,29 \pm 0,93 meses. Já os pacientes com ceratopatia bolhosa foram os que esperaram mais tempo: 9,15 $\pm 6,47$ meses $\left(\chi^{2}=26,5\right.$ e $\mathrm{p}<0,001)$ (Gráfico 2).

\section{DISCUSSÃO}

Ao analisar o número de pacientes que foram inscritos no banco de olhos com indicação de transplante de córnea no estado de Sergipe, no período estudado, observou-se que,

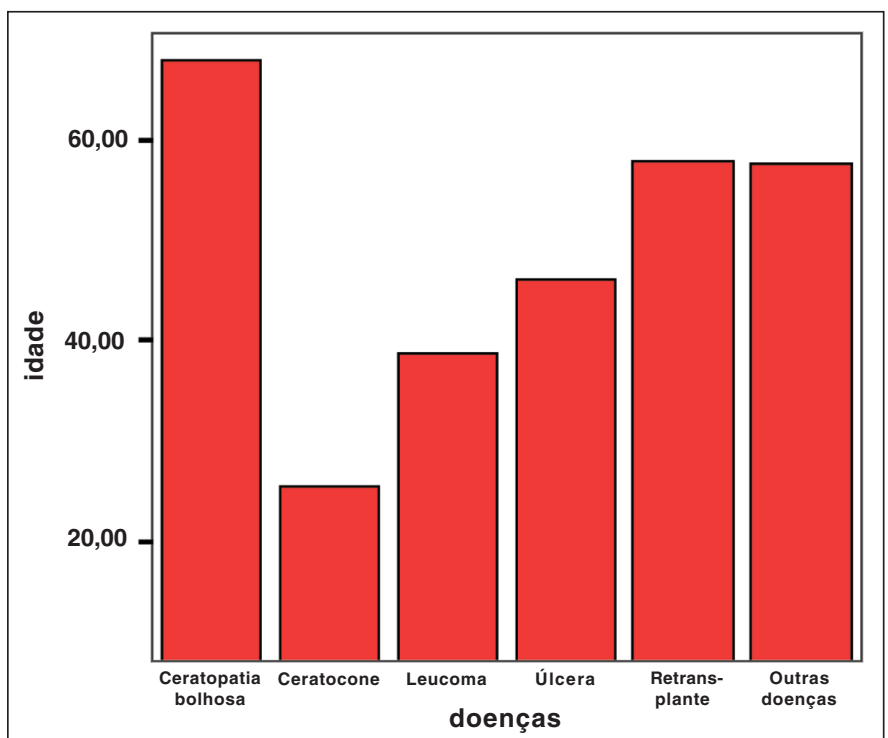

Gráfico 1 - Correlação entre doença corneana e faixa etária média (em anos) dos pacientes. A análise de estatística foi realizada pelo teste de Kruskal-Wallis $\left(\chi^{2}=140,3\right.$ e $\left.p<0,001\right)$

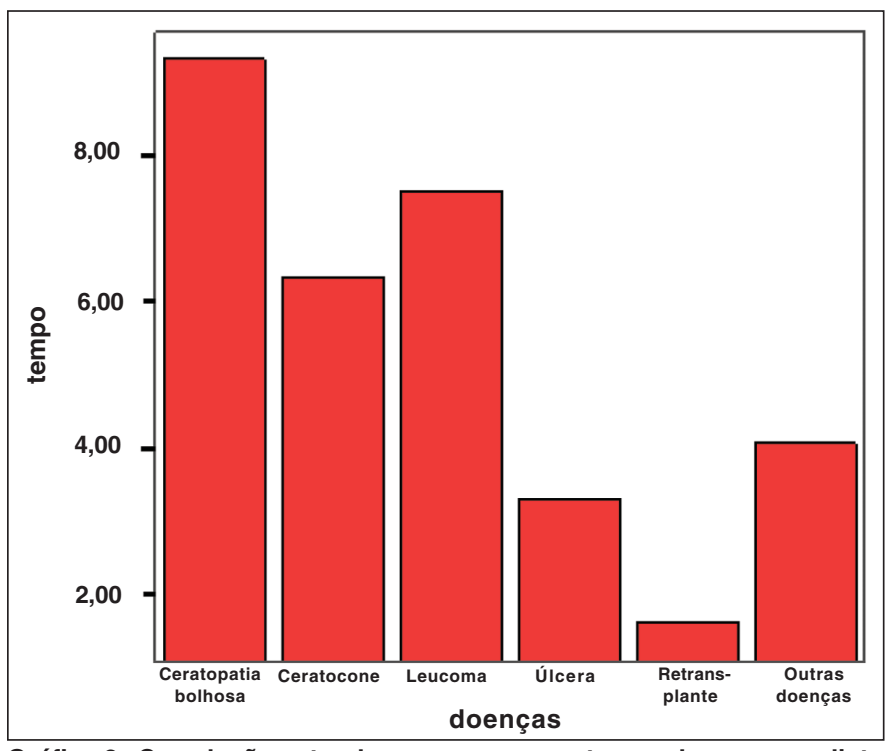

Gráfico 2 - Correlação entre doença corneana e tempo de espera na lista (em meses) até a realização do transplante. A análise de estatística foi realizada pelo teste de Kruskal-Wallis $\left(\chi^{2}=26,5\right.$ e $\left.p<0,001\right)$ diferentemente de outros trabalhos brasileiros ${ }^{(3,6)}$, a CBAP foi a doença que mais freqüentemente levou à indicação de ceratoplastia penetrante, correspondendo a 39,1\% dos casos. Esse dado apresentou-se de acordo com algumas publicações norteamericanas ${ }^{(8,10)}$, cuja percentagem varia de 31,5 a $40,9 \%$. Outros estudos realizados demonstraram que tem ocorrido um decréscimo na indicação de CP por essa desordem ${ }^{(11-12)}$. Contudo, em outro estudo não ocorreram mudanças significantes na indicação de transplante ${ }^{(13)}$, pelo contrário, demonstraram aumento neste tipo de doença levando a intervenção cirúrgica ${ }^{(14)}$.

As outras desordens avaliadas, neste estudo, não demonstraram perfil similar a nenhum outro publicado na literatura. A maioria dos trabalhos apresenta o ceratocone como principal causa ${ }^{(6-7,9)}$. Neste estudo, essa doença ocupou a terceira indicação, precedida por leucoma. Ao analisar o preenchimento do prontuário médico com o diagnóstico de leucoma, verificou-se um grande erro na caracterização das doenças, visto que o leucoma é um sinal e não é um diagnóstico. É a conseqüência final de diversas patologias ${ }^{(15)}$. Neste caso, pode-se indicar dificuldade ocorrida em definir a etiologia do leucoma.

Quando se avaliou a idade dos pacientes e se cruzou com a indicação, verificou-se que os pacientes com indicação a CP tinham idade média de 68,54 anos. Isso permite concluir que a idade acometida está relacionada a complicações decorrentes da facectomia com ou sem implante de lente intra-ocular, podendo isso estar relacionado com vários fatores ${ }^{(15)}$. Considerandose que a cirurgia de catarata tem evoluído muito nas últimas décadas, a avaliação bem detalhada do endotélio corneano associada à melhoria da técnica individual de cada cirurgião, material cirúrgico e um bom acompanhamento clínico pós-operatório poderão diminuir o número de pacientes conduzidos ao banco de olhos ${ }^{(2)}$. Quanto aos pacientes com ceratocone, foram indicados para transplante com idade inferior ao referido na literatura: vinte e três anos neste estudo contra 40 anos nos outros trabalhos ${ }^{(8,10)}$.

A quantidade de pessoas dos sexos masculino e feminino estava de acordo com a maioria dos trabalhos e com a população em geral ${ }^{(2,7-8,16)}$.

Quanto à acuidade visual pré-operatória, variável comumente não descrita nos trabalhos, observou-se que aproximadamente $50 \%$ dos pacientes tinham visão de vultos ou PL, $15 \%$ tinham visão melhor ou igual a 20/400, e 6,6\% melhor ou igual a 20/200. O que mais chamou a atenção foi a quantidade de prontuários sem o preenchimento adequado ou com grande perda visual não caracterizada, atingindo um total de $17,5 \%$ e o grande número de pacientes amauróticos (7,5\%) que estavam inscritos no banco de olhos.

O tempo médio de espera na lista foi de aproximadamente 6 meses. Constatou-se que as desordens que menos tempo esperaram até a conduta cirúrgica foram as que tinham diagnóstico de úlcera de córnea e os casos de retransplante. Considerando-se que a CBAP foi a principal indicação de transplante e foi a doença que mais tempo esperou na fila para transplante (aproximadamente 9,15 meses em média), verifi- 
cou-se que, até a conduta cirúrgica, os pacientes aguardaram muito tempo. Neste aspecto, necessita-se de um grande incremento na quantidade de transplantes a serem realizados, já que, além da acuidade visual reduzida, a ceratopatia bolhosa gera desconforto e dor ocular.

\section{CONCLUSÃO}

Em suma, pode-se concluir que: (1) a ceratopatia bolhosa do afácico e do pseudofácico é a principal indicação para transplante de córnea em nosso meio; (2) não há diferenças entre a freqüência dos sexos; (3) a idade média dos pacientes está de acordo com o aparecimento da doença mais freqüente; (4) a acuidade visual está dentro do esperado; (5) o tempo em lista de espera é longo e inadequado; (6) e é necessário maior atenção dos profissionais durante a indicação do transplante e preenchimento dos prontuários.

\section{AGRADECIMENTOS}

Os autores gostariam de agradecer aos funcionários da Central de Transplantes de Sergipe pela disposição e boa vontade para nos fornecer os dados necessários para esta pesquisa.

\section{ABSTRACT}

Purpose: To define the epidemiological aspects of the patients on the waiting list and those submitted to corneal transplantation in the state of Sergipe, Brazil and the main indications for this procedure. Methods: The data of 320 patients who were on the waiting list and those who had their corneas transplanted from May 2000 to November 2002 were collected at the Transplantation Center of Sergipe. The analyzed data were: corneal disease, age, sex, visual acuity and the time spent on the waiting list until the transplantation was performed. Results: Pseudophakic and aphakic bullous keratopathy was the most common disease (39.1\%), followed by leukoma (22.5\%), keratoconus (14.1\%), “other diseases” (10.6\%), regraft (7.8\%) and ulcerative conditions (5.6\%). Mean age was 52.16 years and there was no significant difference between the sexes. "Hand motion" and "light perception" were the most common visual acuities. The 110 patients who had their cornea transplanted waited, on average, six months. The ones who were indicated for transplant due to ulcerative conditions and regraft had priority and waited less time. Those who had bullous keratopathy were 68.5 years old on average whereas the ones with keratoconus were 23.6 years old. Conclusions: It is possible to conclude that bullous keratopathy is the main indication for corneal transplant in our state. There is no difference in the frequency concerning sex; the mean age of the most common disease is in agreement with the time of its development. Visual acuity is as expected and the time spent on the waiting list is long and inappropriate.

Keywords: Corneal transplantation; Epidemiological profiles; Keratoplasty, penetrating; Keratoconus; Epidemiology

\section{REFERÊNCIAS}

1. Belfort Jr R. Ceratoplastias e ceratectomia. In: Belfort Junior R, Kara José N, editor. Córnea: clínica-cirúrgica. São Paulo: Roca; 1996. p.493-504.

2. Lindquist TD, McGlothan JS, Rotkis WM, Chandler JW. Indications for penetrating keratoplasty: 1980-1988. Córnea. 1991;10(3):210-6.

3. Gonçalves EC, Trindade FC. Ceratoplastia penetrante: alterações nas indicações, 1983-1992. Arq Bras Oftalmol. 1994;57(4):274-7.

4. Thomaz A, Ando E, Akaishi L, Barros AC. Rejeição em transplante de córnea. Rev Bras Oftalmol. 1990;49(6):15-20.

5. Barros CR, Oliveira DF, Castro RS, Lima VMP, Kara José N. Incidência e falência em transplante de córnea [resumo]. Arq Bras Oftalmol. 1992;55(4): 178.

6. Cattani S, Kwitko S, Kroeff MAH, Marinho D, Rymer S, Bocaccio FL. Indicações de transplante de córnea no Hospital de Clínicas de Porto Alegre. Arq Bras Oftalmol. 2002;65(1):95-8.

7. Edwards M, Clover GM, Brookes N, Pendergrast D, Chaulk J, McGhee CN. Indications for corneal transplantation in New Zealand: 1991-1999. Cornea. 2002;21(2):152-5

8. Cosar CB, Sridhar MS, Cohen EJ, Held EL, Alvim P de T, Rapuano CJ et al. Indications for penetrating keratoplasty and associated procedures, 1996-2000. Cornea. 2002;21(2):148-51.

9. Legeais JM, Parc C, d'Hermies F, Pouliquen Y, Renard G. Nineteen years of penetrating keratoplasty in the Hotel-Dieu Hospital in Paris. Cornea. 2001;20 (6):603-6.

10. Dobbins KR, Price FW Jr, Whitson WE. Trends in the indications for penetrating keratoplasty in the midwestern United States. Cornea. 2000;19(6): 813-6.

11. Liu E, Slomovic AR. Indications for penetrating keratoplasty in Canada, 1986-1995. Cornea. 1997;16(4):414-9.

12. Mamalis N, Anderson CW, Kreisler KR, Lundergan MK, Olson RJ. Changing trends in the indications for penetrating keratoplasty. Arch Ophthalmol. 1992;110(10):1409-11.

13. Lois N, Kowal VO, Cohen EJ, Rapuano CJ, Gault JA, Raber IM, Laibson PR. Indications for penetrating keratoplasty and associated procedures, 19891995. Cornea. 1997;16(6):623-9.

14. Ramsay AS, Lee WR, Mohammed A. Changing indications for penetrating keratoplasty in the west of Scotland from 1970 to 1995. Eye. 1997;11(Pt 3): 357-60.

15. Kanski JJ. Oftalmologia clínica: uma abordagem sistêmica. Rio de Janeiro: Revinter; 2004.

16. Endriss D, Cunha F, Ribeiro MP, Toscano J. Ceratoplastias penetrantes realizadas na Fundação Altino Ventura: revisão dos resultados e complicações. Arq Bras Oftalmol. 2003;66(3):273-7.

Nos artigos enviados para publicação, o nome dos autores e suas afiliações devem estar completos. Isso facilitará a indexação e os links com as bases de dados e o CV Lates. 